\title{
Some higher-dimensional vacuum solutions
}

\author{
Metin Gurses"1 and Atalay Karasu ${ }^{2}$ \\ ${ }^{1}$ Department of Mathematics, Faculty of Sciences, Bilkent University, 06533 Ankara, Turkey \\ ${ }^{2}$ Department of Physics, Faculty of Arts and Sciences, Middle East Technical University, \\ 06531 Ankara, Turkey
}

Received 4 August 2000

\begin{abstract}
We study an even-dimensional manifold with a pseudo-Riemannian metric with arbitrary signature and arbitrary dimensions. We consider the Ricci flat equations and present a procedure to construct solutions to some higher(even) dimensional Ricci flat field equations from the four-dimensional Ricci flat metrics. When the four-dimensional Ricci flat geometry corresponds to a colliding gravitational vacuum spacetime our approach provides an exact solution to the vacuum Einstein field equations for colliding gravitational plane waves in an (arbitrary) even-dimensional spacetime. We give explicitly higherdimensional Szekeres metrics and study their singularity behaviour.
\end{abstract}

PACS numbers: 0450, 0420J, 0430

\section{Introduction}

In the general theory of relativity there exist several solution-generating techniques for the vacuum and electrovacuum Einstein field equations [1,2]. These techniques basically allow us to construct metrics from the known metrics. Recently [3,4], we have given a direct construction of the metrics of the $2 \mathrm{~N}$-dimensional Ricci flat geometries from the twodimensional minimal surfaces in a pseudo-Euclidean 3-geometry. In this work we present a procedure to obtain solutions to some higher-dimensional Ricci flat field equations from some four-dimensional Ricci flat metrics. We show that starting from a Ricci flat metric of a four-dimensionalgeometryadmittingtwoKillingvectorfieldsitispossibletogenerateawhole class $2 \mathrm{~N}$-dimensional Ricci flat metrics. Here, in general, both the four-dimensional and 2 Ndimensional geometries have arbitrary signatures. Among these there are some geometries which have physical importance in the general theory of relativity and also in the low-energy limit of string theory. For example, if the four-dimensional geometry describes the colliding gravitationalplane-wavegeometrythenthe $2 \mathrm{~N}$-dimensionalgeometry,forall $\mathrm{N}>2$,describes colliding vacuum gravitational plane waves in the higher-dimensional Einstein theory. We 
give a direct construction of the $2 \mathrm{~N}$-dimensional metrics from the four-dimensional Ricci flat metrics. As an explicit example we give a higher-dimensional extension of the Szekeres [5] colliding vacuum gravitational plane-wave metrics.

0264-9381/01/030509+08\$30.00 C 2001 IOP Publishing Ltd Printed in the UK

509

The singularity structure of these higher-dimensional solutions is examined by using the curvature invariant. It is shown that the singularity becomes weaker or stronger depending upontheparametersofthesolution. Hencethesingularitycharacterofthesolutionmaychange with increasing numbers of dimensions.

Let $\mathrm{M}$ be a $(2 \mathrm{~N}=2+2 \mathrm{n})$-dimensional manifold with a metric ds $2=$

$g_{\alpha \beta} d x \alpha d x \beta$

$$
=g_{a b}\left(x^{c}\right) d x^{a} d x^{b}+H_{A B}\left(x^{c}\right) d y^{A} d y^{B},
$$

where $x^{\alpha}=\left(x^{a}, y^{A}\right), x^{a}$ denote the local coordinates on a two-dimensional manifold and $y^{A}$ denote the local coordinates on a $2 n$-dimensional manifold and $a, b=1,2, A, B=1,2, \ldots, 2 n$. The Christoffel symbols of the metric $g_{\alpha \beta}$ are given by

$$
\begin{aligned}
\Gamma_{B a}^{A} & =\frac{1}{2} H^{A D} H_{D B, a}, \quad \Gamma_{A B}^{a}=-\frac{1}{2} g^{a b} H_{A B, b} \quad, \quad \bar{\Gamma}_{b c}^{a}=\Gamma_{b c,}^{a}, \\
\text { (2) } \Gamma_{B D}^{A} & =\Gamma_{a b}^{A}=\Gamma_{A B}^{a}=0,
\end{aligned}
$$

where the $\Gamma_{b c}^{a}$ are the Christoffel symbols of the two-dimensional metric gab.

The components of the Riemann tensor are given by

$$
R_{\beta \gamma \sigma}^{\alpha}=\Gamma_{\beta \gamma, \sigma}^{\alpha}-\Gamma_{\beta \sigma, \gamma}^{\alpha}+\Gamma_{\rho \gamma}^{\alpha} \Gamma_{\beta \sigma}^{\rho}-\Gamma_{\rho \sigma}^{\alpha} \Gamma_{\beta \gamma}^{\rho} .
$$

The components of the Ricci tensor are

$$
\begin{aligned}
& \mathcal{R}_{a b}=R_{a \alpha b}^{\alpha}=R_{a b}+\frac{1}{4} \operatorname{tr}\left(\partial_{a} H^{-1} \partial_{b} H\right)-\nabla_{a} \nabla_{b} \log \sqrt{\operatorname{det} H}, \\
& \mathcal{R}_{A B}=-\frac{1}{2}\left(g^{a b} H_{A B, b}\right)_{, a}-\frac{1}{2} g^{a b} H_{A B, b}\left[\frac{(\sqrt{\operatorname{det} g})_{, a}}{\sqrt{\operatorname{det} g}}+\frac{(\sqrt{\operatorname{det} H})_{, a}}{\sqrt{\operatorname{det} H}}\right] \\
& +\frac{1}{2} g^{a b} H_{E A, b} H^{E D} H_{D B, a}, \\
& \mathcal{R}_{a A}=0,
\end{aligned}
$$

where $\mathrm{R}_{\mathrm{ab}}$ is the Ricci tensor of the two-dimensional metric $\mathrm{g}_{\mathrm{ab}}$

\section{Ricci flat geometries}

The Ricci flat conditions or the vacuum Einstein field equations are given by

$$
\begin{aligned}
& \partial_{a}\left[\sqrt{\operatorname{det} H g} g^{a b} H^{-1} \partial_{b} H\right]=0, \\
& R_{a b}+\frac{1}{4} \operatorname{tr}\left(\partial_{a} H^{-1} \partial_{b} H\right)-\nabla a \nabla b \log \sqrt{\operatorname{det} H}=0,
\end{aligned}
$$

where $\mathrm{H}$ is a $2 \mathrm{n} \times 2 \mathrm{n}$ matrix of $\mathrm{H}_{\mathrm{AB}}$ and $\mathrm{H}^{-1}$ is its inverse and is the covariant differentiation with respect to the connection $\Gamma_{b c}^{a}$ (or with respect to metric gab). We may rewrite the twodimensional metric as 
where $\eta$ is the metric of flat 2-geometry with arbitrary signature ( 0 or \pm 2 ) and the function $M$ depends on the local coordinates $x^{a}$. The corresponding Ricci tensor and the Christoffel symbol are

$$
\begin{aligned}
& R_{a b}=\frac{1}{2}\left(\nabla_{\eta}^{2} M\right) \eta_{a b}, \\
& \Gamma_{b a}^{c}=\frac{1}{2}\left[-M_{, b} \delta_{a}^{c}-M_{, c} \delta_{b}^{a}+M_{, d} \eta^{c d} \eta_{a b}\right]
\end{aligned}
$$

Now let $\mathrm{H}$ be a block-diagonal matrix of $\mathrm{H}_{\mathrm{AB}}$ and each block is a $2 \times 2$ matrix

$$
\left(\begin{array}{cccc}
\epsilon_{1} \mathrm{e}^{u_{1}} h_{1} & & & \\
& \ddots & & \bigcirc \\
& & \ddots & \\
& & & \epsilon_{n} \mathrm{e}^{u_{i}} h_{i}
\end{array}\right)
$$

with det $h_{i}=1$ and $i= \pm 1$ for all $i=1,2, \ldots, n$. Then

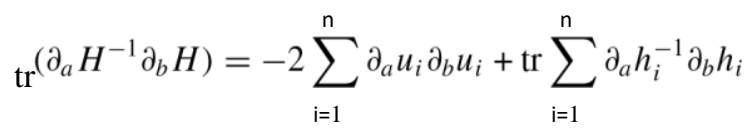

and

$$
\operatorname{det} \mathrm{H}=\mathrm{e}^{2 \mathrm{U}}, \quad \sum_{i=1}^{n} u_{i}=U .
$$

With the above ansatz we can write the higher-dimensional vacuum field equations as $\frac{1}{2} \nabla_{\eta}^{2} M \eta_{a b}-U_{, a b}-\frac{1}{2}\left[M_{, a} U_{, b}+M_{, b} U_{, a}-M_{, d} U_{,}^{d} \eta_{a b}\right]$

$$
-\frac{1}{2} \sum_{i=1}^{n} \partial_{a} u_{i} \partial_{b} u_{i}+\frac{1}{4} \operatorname{tr} \sum_{i=1}^{n} \partial_{a} h_{i}^{-1} \partial_{b} h_{i}=
$$

and

$$
\partial_{a}\left[\eta^{a b} e^{u} \partial_{b} u_{i}\right]=0,
$$

$$
\partial a\left[\eta_{a b e u h}-i 1 \partial b h_{i}\right]=0 \text {, }
$$

(16) where there is no sum over $\mathrm{i}$ (for all $\mathrm{i}=$

$1,2, \ldots, n)$

\section{Four-dimensional geometries}

We first consider the four-dimensional case $(n=1)$. We distinguish the metric functions of the four-dimensional case from the higher-dimensional $(n>1)$ metric functions by letting

$$
M=M, \quad U=U, \quad h=h_{0} .
$$

Since there are infinitely many possible solutions of the vacuum four-dimensional Ricci flat equations we shall denote $\mathrm{Mi}_{\mathrm{i}} \mathrm{h}_{0 \mathrm{i}}, \mathrm{i}=1,2, \ldots, \mathrm{m}$ to distinguish this difference. We label all these different solutions by using a subscript $\mathrm{i}=1,2, \ldots, \mathrm{m}$. Any two different solutions either have different analytic forms or have the same analytic forms but with different integration 
constants. We assume that all of these different solutions have the same metric function U. By this choice we lose no generality because it is a matter of choosing a proper coordinate system. The field equations are

$$
\begin{gathered}
\frac{1}{2}\left(\nabla_{\eta}^{2} \mathcal{M}_{i}\right) \eta_{a b}-\mathcal{U}_{, a b}-\frac{1}{2}\left[\mathcal{M}_{i, a} \mathcal{U}_{, b}+\mathcal{M}_{i, b} \mathcal{U}_{, a}-\mathcal{M}_{i, d} \mathcal{U}_{,}^{d} \eta_{a b}\right] \\
-\frac{1}{2} \partial_{a} \mathcal{U} \partial_{b} \mathcal{U}+\frac{1}{4} \operatorname{tr}\left(\partial_{a} h_{0 i}^{-1} \partial_{b} h_{0 i}\right)=0,
\end{gathered}
$$

and

$$
\begin{aligned}
& \partial_{a}\left[\eta^{a b} e^{U} \partial_{b} U\right]=0, \\
& \partial_{a}\left[\eta_{a b e u h-0 i 1} \partial_{b} h 0 i\right]=0 .
\end{aligned}
$$

For each $i=1,2, \ldots, m$ where $m$ is an arbitrary integer, each triple

$$
\left(\mathrm{Mi}, \mathrm{h}_{0 \mathrm{i}}, \mathrm{U}\right)
$$

forms a solution to the four-dimensional vacuum field equations and we assume that the function $U$, for all of these different solutions, is the same.

\section{Higher-dimensional Ricci flat geometries}

We start with the assumptions that $U=U$ where the function $U$ is defined in (13), $h_{i}=h_{o i}$ and $\mathrm{m}=\mathrm{n}$ and using (18) into (14) we obtain

$$
\begin{gathered}
\frac{1}{2} \nabla_{\eta}^{2}(M-\tilde{M}) \eta_{a b}+(n-1) \mathcal{U}_{, a b}-\frac{1}{2}\left[(M-\tilde{M})_{, a} \mathcal{U}_{, b}+(M-\tilde{M})_{, b} \mathcal{U}_{, a}-(M-\tilde{M})_{, d} \mathcal{U}_{,}^{d} \eta_{a b}\right] \\
-\frac{1}{2} \sum_{i=1}^{n} \partial_{a} u_{i} \partial_{b} u_{i}+\frac{1}{2} n \partial_{a} \mathcal{U} \partial_{b} \mathcal{U}=0,
\end{gathered}
$$

where $\sum_{i=1}^{n} \mathcal{M}_{i}=\tilde{M}$. Define $\mathrm{M}-\mathrm{M}^{\sim}=\mathrm{M}^{-}$, the above equation can be written as

$$
\begin{aligned}
\frac{1}{2}\left(\nabla_{\eta}^{2} \bar{M}\right) \eta_{a b}+ & (n-1) \mathcal{U}_{, a b}-\frac{1}{2}\left[\bar{M}_{, a} \mathcal{U}_{, b}+\bar{M}_{, b} \mathcal{U}_{, a}-\bar{M}_{, d} \mathcal{U}_{,}^{d} \eta_{a b}\right] \\
& -\frac{1}{2} \sum_{i=1}^{n} \partial_{a} u_{i} \partial_{b} u_{i}+\frac{1}{2} n \partial_{a} \mathcal{U} \partial_{b} \mathcal{U}=0
\end{aligned}
$$

We assume that $U, h_{0 i}$ for $i=1,2, \ldots, n$ are given functions of $x^{a}$. Hence given $U$ we can solve (15) for $u_{i}$ with $i=1,2, \ldots, n$, or

$$
\stackrel{2}{\nabla \eta u_{i}+\eta U_{,} a u_{i, b}}=0 .
$$

Then inserting $\mathrm{U}, \mathrm{u}_{i}$ and $\mathrm{h}_{\mathrm{oi}}$ in $(22)$ we solve the function $\mathrm{M}^{-}$. Then we have the following theorem.

Theorem. If ${ }_{\mathrm{U}, \mathrm{h}_{\mathrm{oi}}}$ and $\mathrm{Mi}$, for each $\mathrm{i}=1,2, \ldots, \mathrm{n}$, form a solution to the four-dimensional Ricci flat field equations for the metric

$$
d s^{2}=e^{-M_{i}} \eta_{a b} d x^{a} d x^{b}+e^{U}\left(h_{0 i}\right)_{a b} d y^{a} d y^{b}, i=1,2, \ldots, n,(24) \text { where } M_{i}=M_{i}\left(x^{a}\right), U=
$$

$\mathrm{U}\left(\mathrm{x}^{\mathrm{a}}\right)$ and $\mathrm{h}_{0 \mathrm{i}}=\mathrm{h}_{0 \mathrm{i}}\left(\mathrm{x}^{\mathrm{a}}\right)$, then the metric of the $(2 \mathrm{n}+2)-$ 
dimensional geometry is defined below

$$
\mathrm{d}^{s^{2}}=\mathrm{e}^{-M} \eta_{a b} \mathrm{~d} x^{a} \mathrm{~d} x^{b}+\sum_{i=1}^{\mathrm{n}} \epsilon_{i} \mathrm{e}^{u_{i}}\left(h_{0 i}\right)_{a b} \mathrm{~d} y_{i}^{a} \mathrm{~d} y_{i}^{b},
$$

solves the Ricci flat equations, where $\epsilon_{i}= \pm 1, M=\bar{M}+\tilde{M}, \tilde{M}=\sum_{i=1}^{n} \mathcal{M}_{i}, \bar{M}_{\text {solves (22) }}$ and $\mathrm{u}_{\mathrm{i}}$ solve (23). Here the local coordinates of the $(2 n+2)$-dimensional geometry are given by $x^{\alpha}=\left(x^{a}, y_{1}^{a}, y_{2}^{a}, \ldots, y_{n}^{a}\right)$.

We shall now consider some examples which will be obtained by the application of the theorem. We shall consider the case which has a physical importance as far as Einstein's theory of general relativity is concerned. We let $i=1$ for all $i=1,2, \ldots, n$ and

$$
\eta=\left(\begin{array}{ll}
0 & 1 \\
1 & 0
\end{array}\right), \quad x^{1}=u, \quad x^{2}=v,
$$

then the equations in (22) become

$$
\begin{aligned}
& \partial_{u} \bar{M} \partial_{u} \mathcal{U}=(n-1) \partial_{u u} \mathcal{U}-\frac{1}{2} \sum_{i=1}^{n}\left(\partial_{u} u_{i}\right)^{2}+\frac{1}{2} n\left(\partial_{u} \mathcal{U}\right)^{2} \\
& \partial_{v} \bar{M} \partial_{v} \mathcal{U}=(n-1) \partial_{v v} \mathcal{U}-\frac{1}{2} \sum_{i=1}^{n}\left(\partial_{v} u_{i}\right)^{2}+\frac{1}{2} n\left(\partial_{v} \mathcal{U}\right)^{2},
\end{aligned}
$$

where the (uv) component of (22) is satisfied identically by virtue of equations (26), (27), (23) and (19). The above equations remind us of the construction of the solutions of the EinsteinMaxwell-massless scalar field equations from the metrics of the Einstein-Maxwell spacetimes [8]. Equation (23) becomes

$$
2 u_{i, u v}+U, u u i, v+U, v u i, u=0 .
$$

Hence for all $n>1$ to find a solution of higher-dimensional colliding gravitational vacuum plane waves we have to solve the above equations (26)-(28) for $\mathrm{M}^{-}$and $\mathrm{u}_{i}, \mathrm{i}=1,2, \ldots, \mathrm{n}$. We shall now make a further assumption which solves (28) identically. Let $u_{i}=m_{i} U$ where $m_{i}(i=$ $1,2, \ldots, n)$ are real constants satisfying only the condition

$$
\sum_{i=1}^{n} m_{i}=1
$$

otherwise they are arbitrary. Then the solution of (26) and (27) can be found as

$$
e^{-} M^{-}=(f u g v)-n+1(f+g)(m 2+n-2) / 2 .
$$

Here we took

$$
e^{u}=f(u)+g(v)
$$

whichisthegeneralsolutionof(19)wheref(u)andg(v)arearbitrary(differentiable)functions of $u$ and $\mathrm{v}$, respectively, and

$$
\sum_{i=1}^{n}\left(m_{i}\right)^{2}=m^{2} .
$$


Hence according to our theorem given above this completes the construction of the metric of the corresponding vacuum spacetimes of dimension $2 n+2$. Given any four-dimensional metric of colliding vacuum gravitational plane-wave geometry (see [6] for details) we have their extensions to higher dimensions for arbitrary $n$ without solving any further differential equations. Sometimes to avoid some undesired singularities on the whole $(2 n+2)$-dimensional geometry it may be necessary to keep all the integration constants of the original fourdimensional metric variables $\left(\mathrm{Mi}_{\mathrm{i}} \mathrm{U}, \mathrm{h}_{0 \mathrm{i}}\right)$. The boundary conditions discussed in [5] and in [6,chapter 7, pp 46-7] of the four-dimensional metrics should be used for the functions Mi to make them continuous across the boundaries $u=0, v=0$. Rather we have to use them to make the $(2 n+2)$-dimensional metric function $M$ continuous across these boundaries.

\section{Higher-dimensional Szekeres solution}

For illustration let us take the Szekeres solutions [5,6] (which contains the Khan-Penrose [7] solution as a special case) as the four-dimensional vacuum solutions. They are given by

$$
d s^{2}=2 e^{-M_{i}} d u d v+e^{U-v_{i}} d x^{2}+e^{U+v_{i}} d y^{2}, \quad i=1,2, \ldots, n
$$

where

$$
\begin{gathered}
V_{i}=-2 k_{i} \tanh ^{-1}\left(\frac{\frac{1}{2}-f}{\frac{1}{2}+g}\right)^{1 / 2}-2 \ell_{i} \tanh ^{-1}\left(\frac{\frac{1}{2}-g}{\frac{1}{2}+f}\right)^{1 / 2}, \\
\mathcal{M}_{i}=-\log \left(c_{i} f_{u} h_{v}\right)-\frac{1}{2}\left(k_{i}^{2}+\ell_{i}^{2}+2 k_{i} \ell_{i}-1\right) \log (f+g) \\
+\frac{1}{2} k_{i}^{2} \log \left(\frac{1}{2}-f\right)+\frac{1}{2} \ell_{i}^{2} \log \left(\frac{1}{2}-g\right)+\frac{1}{2} \ell_{i}^{2} \log \left(\frac{1}{2}+f\right)+\frac{1}{2} k_{i}^{2} \log \left(\frac{1}{2}+g\right) \\
+2 k_{i} \ell_{i} \log \left(\sqrt{\frac{1}{2}-f} \sqrt{\frac{1}{2}-g}+\sqrt{\frac{1}{2}+f} \sqrt{\frac{1}{2}+g}\right),
\end{gathered}
$$

where $k_{i}, i$ and $c_{i}$ are constants for all $i=1,2, \ldots, n$, and

$$
f=\frac{1}{2}-\left(e_{1} u\right)^{n_{1}}, \quad g=\frac{1}{2}-\left(e_{2} v\right)^{n_{2}} .
$$

Here $e_{1}, e_{2}, n_{1} \geqslant 2$, and $n_{2} \geqslant 2$ are also arbitrary constants. To avoid the discontinuity of the function $e^{-M_{i}}$ along the boundaries $u=0$ and $v=0$ some relations among $k_{i, j}$ and $n_{1}, n_{2}$ are needed. We shall not set these relations, because in our case the continuity of the function $\mathrm{e}^{-\mathrm{M}}$ is important. For this purpose we give similar relations among these constants. Let us first define

$$
k^{2}=\sum_{i=1}^{n} k_{i}^{2}, \quad \ell^{2}=\sum_{i=1}^{n} \ell_{i}^{2}, \quad s=\sum_{i=1}^{n} k_{i} \ell_{i}
$$

and let

$$
k^{2}=2\left(1-\frac{1}{n_{1}}\right), \quad \ell^{2}=2\left(1-\frac{1}{n_{2}}\right),
$$

where $\mathrm{n}_{1} 2, n_{2} \geqslant 2$. We observe that the constants $\mathrm{k}$ and, are restricted to the range satisfying

$$
1 \leqslant k^{2}<2, \quad 1 \leqslant \ell^{2}<2
$$

It is now easy to calculate $M$ which is continuous across the boundaries $u=0$ and $v=0$ (by virtue of the conditions (38)). It reads 


$$
\mathrm{e}^{-M}=\frac{(f+g)^{\left(k^{2}+\ell^{2}+m^{2}+2 s-2\right) / 2}}{\left(\frac{1}{2}+f\right)^{k^{2} / 2}\left(\frac{1}{2}+g\right)^{\ell^{2} / 2}\left(\sqrt{\frac{1}{2}-f} \sqrt{\frac{1}{2}-g}+\sqrt{\frac{1}{2}+f} \sqrt{\frac{1}{2}+g}\right)^{2 s}} .
$$

We also set $\Pi_{i=1}^{n} c_{i}=\left(e_{1} e_{2} n_{1} n_{2}\right)^{-1}$. Hence the metric of the $(2 \mathrm{n}+2)$-dimensional spacetime becomes

$$
\mathrm{d}^{s^{2}}=2 \mathrm{e}^{-M} \mathrm{~d} u \mathrm{~d} v+\sum_{\mathrm{i}=1}^{\mathrm{n}}(f+g)^{m_{i}}\left(\mathrm{e}^{-V_{i}} \mathrm{~d} x_{i}^{2}+\mathrm{e}^{V_{i}} \mathrm{~d} y_{i}^{2}\right),
$$

where $m_{i}, i=1,2, \ldots, n$ are constants with the condition given in (29) and $V_{i}$ are given in (34). Here $\mathrm{x}_{1}=\mathrm{x}, \mathrm{y}_{1}=\mathrm{y}$. When $\mathrm{n}=1$ we have $\mathrm{m}_{1}=1, \mathrm{~m}=1, \mathrm{~s}=\mathrm{k}$, which corresponds to the fourdimensional case.

\section{Curvature singularities}

Next, wecalculatethecurvatureinvariantofthemetric(1). ThecomponentsoftheRiemannian tensor are

$$
\begin{aligned}
& R_{B a b}^{A}=\Gamma_{B b, a}^{A}-\Gamma_{B a, b}^{A}+\Gamma_{D a}^{A} \Gamma_{B b}^{D}-\Gamma_{D b}^{A} \Gamma_{B a}^{D} \\
& R_{a b B}^{A}=\Gamma_{a B, b}^{A}+\Gamma_{D b}^{A} \Gamma_{a B}^{D}-\Gamma_{c B}^{A} \Gamma_{a b}^{c}, \\
& R_{b A B}^{a}=\Gamma_{D A}^{a} \Gamma_{b B}^{D}-\Gamma_{E B}^{a} \Gamma_{b A}^{E}, \\
& R_{b c d}^{a}=\Gamma_{b d, c}^{a}-\Gamma_{b c, d}^{a}+\Gamma_{e c}^{a} \Gamma_{b d}^{e}-\Gamma_{e d}^{a} \Gamma_{b c}^{e}, \\
& R_{B D E}^{A}=\Gamma_{a D}^{A} \Gamma_{B E}^{a}-\Gamma_{a E}^{A} \Gamma_{B D}^{a}, \\
& R_{B D b}^{A}=0, \quad R_{a b c}^{A}=0 .
\end{aligned}
$$

The curvature invariant is defined by

$$
I=R_{\mu v \alpha \beta} R_{\mu v \alpha \beta} \text {. }
$$

This can be written as

$$
I=R a b c d R a b c d+R A B D E R A B D E+2 R A B a b R A B a b+4 R_{a A b B R} a A b B,
$$

$$
\begin{aligned}
& \text { where } \\
& \begin{aligned}
R^{a b c d} R_{a b c d}= & R^{2}, \\
R^{A B C D} R_{A B C D} & =\frac{1}{8} g^{a b} g^{c d}\left[\operatorname{tr}\left(\partial_{c} H^{-1} \partial_{a} H\right) \operatorname{tr}\left(\partial_{d} H^{-1} \partial_{b} H\right)-\operatorname{tr}\left(\partial_{c} H^{-1} \partial_{b} H \partial_{d} H^{-1} \partial_{a} H\right)\right] \\
R^{A B a b} R_{A B a b}= & \frac{1}{8} g^{a b} g^{c d}\left[\operatorname{tr}\left(\partial_{a} H^{-1} \partial_{d} H \partial_{c} H^{-1} \partial_{b} H\right)-\operatorname{tr}\left(\partial_{b} H^{-1} \partial_{c} H \partial_{a} H^{-1} \partial_{d} H\right)\right], \\
R^{a A b B} R_{a A b B}= & \frac{1}{4} g^{a d} g^{e b} \operatorname{tr}\left(H^{-1} \nabla_{a} \nabla_{b} H H^{-1} \nabla_{d} \nabla_{e} H\right) \\
& +\frac{1}{4} g^{a d} g^{e b} \operatorname{tr}\left(H^{-1} \nabla_{a} \nabla_{b} H H^{-1} \partial_{d} H H^{-1} \partial_{e} H\right) \\
& +\frac{1}{16} g^{a d} g^{e b} \operatorname{tr}\left(H^{-1} \partial_{b} H H^{-1} \partial_{d} H H^{-1} \partial_{a} H H^{-1} \partial_{e} H\right) .
\end{aligned}
\end{aligned}
$$

We may, in general, discuss the singularity structure of colliding gravitational plane waves in $2 n+2$ dimensions, but the higher-dimensional Szekeres vacuum solutions give a similar feature of this problem. First of all the solutions have delta function curvature singularities across the boundaries $u=0, v=0$ or $f=\frac{1}{2}$ and $g=\frac{1}{2}$ when $\mathrm{n}_{1}=\mathrm{n}_{2}=2$. For other values of $\mathrm{n}_{1}>2$ and $\mathrm{n}_{2}>2$ the curvature has a Heaviside step function discontinuity across these boundaries. In addition to these discontinuities across the boundaries the spacetime has an 
essential singularity on the surface $f(u)+g(v)=0$. For this purpose we shall find the form of the curvature invariant $I$ as $f+g \rightarrow 0$, which is the singular surface for the four-dimensional case. We find that

$$
I \sim\left(f_{u} g_{v}\right)^{2}(f+g)^{-\mu}
$$

where $\mu=k^{2}+,^{2}+m^{2}+2 s+2$. For the four-dimensional case $(n=1)$ let us choose $k=k^{-}{ }_{1,}=$ $,-1, \mathrm{~m}_{1}=1$ and $\mathrm{m}^{2}=1$. Hence in this case $\mu=\bar{k}_{1}^{2}+\bar{\ell}_{1}^{2}+2 \bar{k}_{1} \bar{\ell}_{1+3}$. We have

both $1 \leqslant k^{2} \quad \leqslant \ell^{2} \leqslant k^{2} \quad \ell<2,1<2$ and $1_{1}^{-}<2,1 \leqslant{ }_{1}^{-2}<2$. Hence the constant $m$ plays an important role in the higher-dimensional metrics. On the constants $m_{i}, i=1,2, \ldots, n$ we only have the restriction (29). Hence as $f+g \rightarrow 0$ we obtain

$$
\frac{I_{2 n+2}}{I_{4}} \sim(f+g)^{1-m^{2}-2 s+2 \bar{k}_{1} \bar{\ell}_{1}} .
$$

Here we have made use of conditions (38) for $\mathrm{k}$ and , and exactly similar conditions on $\mathrm{k}^{-} 1$ and

, 1 which imply that $k^{2}=\bar{k}_{1}^{2}=2\left(1-1 / n_{1}\right)$ and $\ell^{2}=\bar{\ell}_{1}^{2}=2\left(1-1 / \mathrm{n}_{2}\right)$. This means that the singularity structure in the higher-dimensional spacetimes can be made weaker and stronger than the four-dimensional cases by choosing the constants $m_{i}, k_{i}$ and ,i properly. We have enough freedom to do this for higher values of $n$.

\section{Conclusion}

We have studied some Ricci flat geometries with arbitrary signatures. We proved a theorem saying that to all Ricci flat metrics of four-dimensional pseudo-Riemannian geometries admitting two Killing vector fields there corresponds a class of Ricci flat metrics for some $(2 n+2)$-dimensional pseudo-Riemannian geometries. As an application we presented an explicitconstructionof $(2 n+2)$-dimensionalmetricsofcollidinggravitationalwavespacetimes from given four-dimensional metrics. We gave a higher-dimensional generalization of the Szekeres metrics and discussed the singularity structure of the corresponding spacetimes. Further construction of higher-dimensional colliding gravitational plane-wave metrics will be communicated elsewhere. A possible extension of our work to the low-energy limit of string theory is possible for an arbitrary n. Another application of our approach presented here may be to the colliding gravitational plane-wave problem for the Einstein-Maxwell-dilaton field equations [9].

\section{Acknowledgments}

This work is partially supported by the Scientific and Technical Research Council of Turkey (TUBITAK) and by the Turkish Academy of Sciences (TUBA).

\section{References}

[1] Kramer D, Stephani H, Mac Callum M A H and Herlt E 1980 Exact Solutions of Einstein's Field Equations (Cambridge: Cambridge University Press)

[2] Hoenselaers C and Dietz W (ed) 1984 Solutions of Einstein's Equations: Techniques and Results (Lecture Notes in Physics no 205) (Berlin: Springer) 
[3] Gurses M 2000 Some Ricci flat pseudo Riemannian geometries" Preprint math. DG/0006040

[4] Gurses M 2000 Sigma models, minimal surfaces and some Ricci flat pseudo Riemannian geometries" Geometry,

Integrability and Quantization (Varna, 7-15 July 2000) ed I M Mladenov and G Naber

[5] Szekeres P 1972 J. Math. Phys. 13286

[6] Griffiths J B 1991 Colliding Plane Waves in General Relativity (Oxford: Clarendon)

[7] Khan K A and Penrose R 1971 Nature 229185

[8] Eris, A and Gurses M 1977" J. Math. Phys. 181303

[9] Gurses M and Sermutlu E 1995“ Phys. Rev. D 52809 